\title{
Novel Mass Spectral Fragmentation of Heptafluorobutyryl Derivatives of Acyl Analogues of Platelet-Activating Factor
}

\author{
Susan T. Weintraub and R. Neal Pinckard \\ Department of Pathology, The University of Texas Health Science Center at San Antonio, San Antonio, \\ Texas, USA
}

Timothy G. Heath and Douglas A. Gage

Department of Biochemistry, Michigan State University, East Lansing, Michigan, USA

\begin{abstract}
Direct derivatization of the acyl analogue of platelet-activating factor (acyl-PAF) with heptafluorobutyric anhydride results in replacement of the phosphocholine moiety with a heptafluorobutyryl (HFB) group. Electron capture (EC) mass spectrometric analysis of this compound that makes use of negative ion detection along with subsequent accurate mass measurement and tandem mass spectrometry studies revealed that in addition to expected fragmentation due to losses of elements of $\mathrm{HF}$, ketene, and/or acetic acid, there is a rearrangement reaction between the HFB group and the substituent on carbon-2 of the glycerol backbone. For 2-acetyl isomers, this fragmentation yields a characteristic ion at $\mathrm{m} / \mathrm{z} 237$; for 1-acetyl isomers, the analogous ion is observed at [M-135] ${ }^{-}$, along with a corresponding carboxylate anion. The use of the HFB derivative is invaluable for analysis of PAF homologues and analogues because it provides detailed structural information in combination with the high sensitivity of a gas chromatugraphy combined with EC-mass spectrometry assay. (J Am Soc Mass Spectrom 1991, 2, 476-482)
\end{abstract}

$\mathrm{P}$ latelet-activating factor (PAF) denotes a unique autacoid class of $s n$-2-acetylated phospholipids (1-O-alkyl-2-acetyl-sn-glycero-3-phosphocholine, AGEPC). Because of its high potency and remarkably wide spectrum of biological activities, PAF has been implicated in the pathogenesis of numerous human diseases [1]. The 16-carbon alkyl chain homologue (C16:0-AGEPC) shown in Figure 1a has been considered to be the most abundant molecular species of PAF synthesized by stimulated human neutrophils. However, more recent studies indicate that at least $50 \%$ of the choline-containing acetylated phosphoglycerides produced by stimulated human neutrophils are 1-acyl-2-acetyl-sn-glycero-3-phosphocholines (AGPC) [2], such as C16:0-AGPC shown in Figure $1 \mathrm{~b}$.

Currently, platelet bioassay is the most widely used method to detect and quantitate PAF; however, the data obtained from platelet bioassay are not valid if the samples contain endogeneous PAF inhibitors. More important, PAF molecular heterogeneity precludes accurate quantitation by bioassay because the numerous alkyl- and acyl-PAF homologues and analogues have different platelet-stimulating potencies

Address reprint requests to Susan $T$. Weintraub, Department of Pathology. The University of Texas Health Science Center at San Antonio, 7703 Floyd Curl Drive, San Antonio, TX 78284-7750.

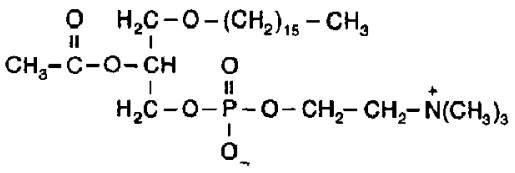

a

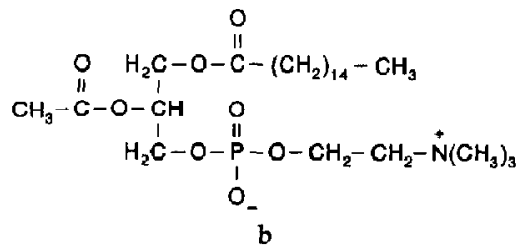

Figure 1. Chemical structures of (a) 1-O-hexadecyl-2-acetyl-snglycero-3-phosphocholine (C16:0-AGEPC) and (b) 1-hexadecanoyl-2-acetyl-sn-glycero-3-phosphocholine (C16:0-AGPC).

[1]. Thus, it is essential to identify each molecular species of PAF in a sample of unknown composition, and mass spectrometry currently provides the only means to accomplish this objective. However, most of the mass spectrometric procedures in the literature have problems in this regard; they either lack the required sensitivity or they are unable to provide structural information. We have developed a novel, highly sensitive gas chromatography combined with 
mass spectrometry (GC/MS) procedure which permits facile identification of the various molecular species of PAF [3]. The procedure relies on the parallel GC/MS analysis of heptafluorobutyryl (HFB) and pentafluorobenzoyl (PFB) derivatives obtained by direct chemical derivatization of PAF. Thus, without requiring either phospholipase $\mathrm{C}$ or $\mathrm{HF}$ treatment, volatile derivatives suitable for GC/MS analysis can readily be prepared. This approach has recently been shown to be very effective for the analysis of PAF obtained from human polymorphonuclear leukocytes [4] and does not result in isomerization of the derivatized product [3].

This same procedure has now been applied to the analysis of acyl-PAF analogues, i.e., AGPC. The current report describes elucidation of a novel fragmentation pathway observed in the electron capture (EC) mass spectrum of the HFB derivative of AGPC and explains its utility for structural elucidation of and differentiation between various molecular species of alkyl- and acyl-PAF homologues and analogues.

\section{Experimental}

Preparation of 1-hexadecanoyl-2-acetyl-sn-glycero3-phosphocholine (C16:0-AGPC)

To a suspension of 1-hexadecanoyl-sn-glycera-3-phosphocholine (lyso-GPC, Bachem Fine Chemicals, Torrence, CA) in $200 \mu \mathrm{L}$ of chloroform was added $100 \mu \mathrm{L}$ of acetic anhydride followed by $5 \mu \mathrm{L}$ of perchloric acid. The reaction was allowed to proceed for $-5 \mathrm{~s}$, and the mixture was then cooled to $0{ }^{\circ} \mathrm{C}$. The product was extracted by the procedure of Bligh and Dyer [5] and purified by thin-layer chromatography using a solvent system of chloroform/methanol/water (65:35:6, vol/vol/vol) [6]. The purified product was characterized by phosphorus analysis [7] and fast atom bombardment (FAB) mass spectrometry.

\section{Derizatization}

In accordance with our previously published procedure for derivatization of up to $10 \mu \mathrm{g}$ of phospholipid $[3,4]$, a chloroform solution of AGPC or AGEPC was dried under a stream of nitrogen in a $10-\mathrm{mL}$ tube, and $100 \mu \mathrm{L}$ of heptafluorobutyric anhydride was added. The tube was sealed with a Teflon-lined screw-cap and incubated at $40^{\circ} \mathrm{C}$ for $4 \mathrm{~h}$. The solvent was then evaporated by a stream of nitrogen, and the residue was partitioned between $400 \mu \mathrm{L}$ of hexane and $400 \mu \mathrm{L}$ of water. The water layer was washed twice with 200 $\mu \mathrm{L}$ of hexane; the organic phases were combined, dried under a stream of nitrogen, and redissolved in hexane prior to injection into the gas chromatograph. For convenience, in the present report the product of derivatization will be named according to the starting phosphocholine and the derivatizating agent, for example, C16:0-AGPC/HFB.

\section{Instrumentation}

Gas chromatography combined with mass spectrometry analyses were performed on a Finnigan-MAT model 4615 mass spectrometer (San Jose, CA) in combination with an INCOS data system. Methane at 0.5 torr was used as the chemical ionization reagent gas for both positive ion chemical ionization (PICI) and $\mathrm{EC}$ with negative ion detection spectra. The ion source temperature was $100^{\circ} \mathrm{C}$, and the electron energy was $70 \mathrm{eV}$. Gas chromatographic separation was accomplished by means of a $15-\mathrm{m} \times 0.32-\mathrm{mm}$ DB-1 fused silica capillary column (J \& W Scientific, Rancho Cordova, CA), which was connected directly to the mass spectrometer via a heated transfer line. The linear velocity of helium was $75 \mathrm{~cm} / \mathrm{s}$, and the injector temperature was $250^{\circ} \mathrm{C}$. A "splitless" injection technique was employed. The column temperature was initially maintained for $1 \mathrm{~min}$ at $180^{\circ} \mathrm{C}$ and was then increased to $225^{\circ} \mathrm{C}$ at a rate of $30^{\circ} \mathrm{C} / \mathrm{min}$.

Accurate mass analyses were performed on a double-focusing mass spectrometer (JEOL HX-110, JEOL USA, Peabody, MA) at a resolution of $10,000(10 \%$ valley) operating under methane EC-MS conditions. Samples were introduced by means of a direct probe inlet. Mass measurements were made by peak matching to ions of known composition in PFK.

Product ion spectra were obtained on a FinniganMAT TSQ70 triple-quadrupole mass spectrometer. Ammonia was employed as the reagent gas, and sample introduction was through the gas chromatograph. A collision energy offset of $20 \mathrm{eV}$ in the laboratory frame of reference was used to induce precursor ion fragmentation. Argon was used as the collision gas in $\mathrm{Q}_{2}$ at a pressure between 0.4 and $0.6 \mathrm{mtorr}$.

Fast atom bombardment mass spectra were acquired on a Finnigan-MAT model 212 double-focusing mass spectrometer in combination with an INCOS data system. A saddle field atom gun (Ion Tech, Middlesex, UK) was used to generate a xenon beam at $8 \mathrm{keV}$. The ion source temperature was $-70^{\circ} \mathrm{C}$, and the accelerating voltage was $3 \mathrm{kV}$. Samples were dissolved in methanol, and $-1 \mu \mathrm{L}$ of the solution was applied to the copper probe tip. Thinglycerol $\left(1-2{ }_{\mu} \mathrm{L}\right)$ was then added and mixed with the sample. The contributions from the matrix were subtracted from each spertrum by means of the data system.

\section{Results}

Reaction of C16:0-lyso-GPC with acetic anhydride in the presence of perchloric acid rapidly and quantitatively produced the C16:0-AGPC used in the present study. From the FAB mass spectrum of this compound (data not shown) it can be determined that the fragmentation of C16:0-AGPC is analogous to that of AGEPC [8], exhibiting intense ions for $[\mathrm{MH}]^{+}(m / z$ 538) and $\mathrm{m} / \mathrm{z} 184$ (representative of the phospho- 
choline moiety) and a low intensity ion at $\mathrm{m} / \mathrm{z} 496$ generated by the loss of the elements of ketene from the protonated molecular species. Unlike the spectrum of C16:0-AGEPC, ions at $m / z 355$ (5\% relative intensity, $\left.[\mathrm{MH}-\text { phosphocholine }]^{+}\right)$and $\mathrm{m} / z 300(10 \%$ relative intensity, $[\mathrm{MH}-238]^{+}$, comparable to the ketene loss described above, but for the hexadecanoyl group instead of the acetyl group) were also observed.

Initially, $\mathrm{CI}$ mass spectra of the HFB derivatives were obtained by using a relatively limited scan range in anticipation of minimal fragmentation due to the ionization conditions. For the HFB derivative of C16:0-AGPC, the negative ion spectrum exhibited ions at $m / z 548,528,488,486$, and 433 . When ammonia was used as the reagent gas a low intensity ion at $m / z 567\left([\mathrm{M}-\mathrm{H}]^{-}\right)$was also seen. With positive ion detection, the base peak was observed at $\mathrm{m} / \mathrm{z} 509$ ([MH-acetic acid $]^{+}$), while $[\mathrm{MH}]^{+}$at $m / z 569$ had a relative intensity of $-10 \%$. Comparable results were obtained for the C18:0 and C18:1-AGPC homologues, with the ions listed above increased by 28 and 26 mass units, respectively. Examination of reconstructed total ion current chromatograms for both positive and negative ion detection revealed that there were actually two closely eluting GC peaks for each derivative (denoted as peaks I and II). The selected ion retrieval traces shown in Figure 2 for C16:0 AGPC/HFB indicated that the components were structurally related but had obvious differences in their mass spectra, suggesting a pair of positional isomers.

Wider scan range $\mathrm{CI}$ spectra were then acquired, as shown in Figure 3a, with the corresponding selected

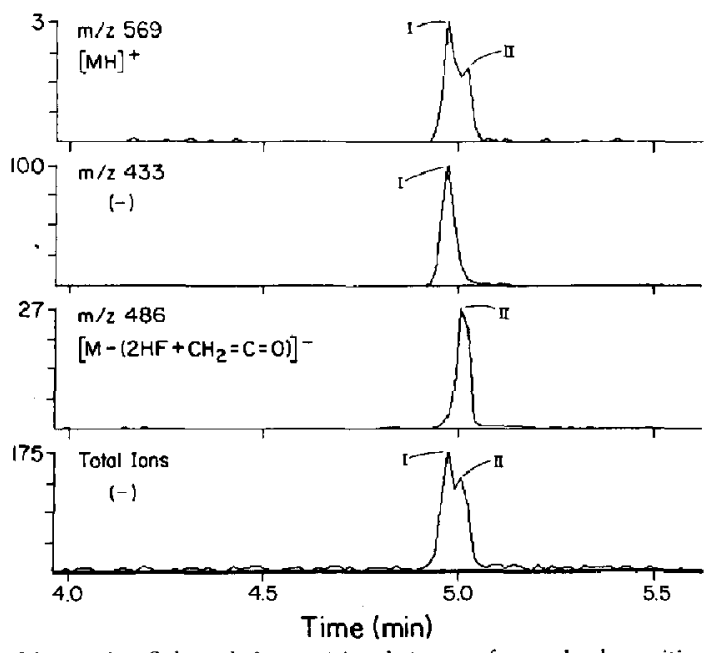

Figure 2. Selected ion retrieval traces for pulsed positive ion/negative ion detection CI GC/MS analysis of the HFB

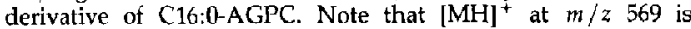
clearly exhibited by both GC peaks while the ion at $m / z 433$ is only associated with the earlier eluting peak (I) and the ion at $m / z 486$ is only seen in peak II.
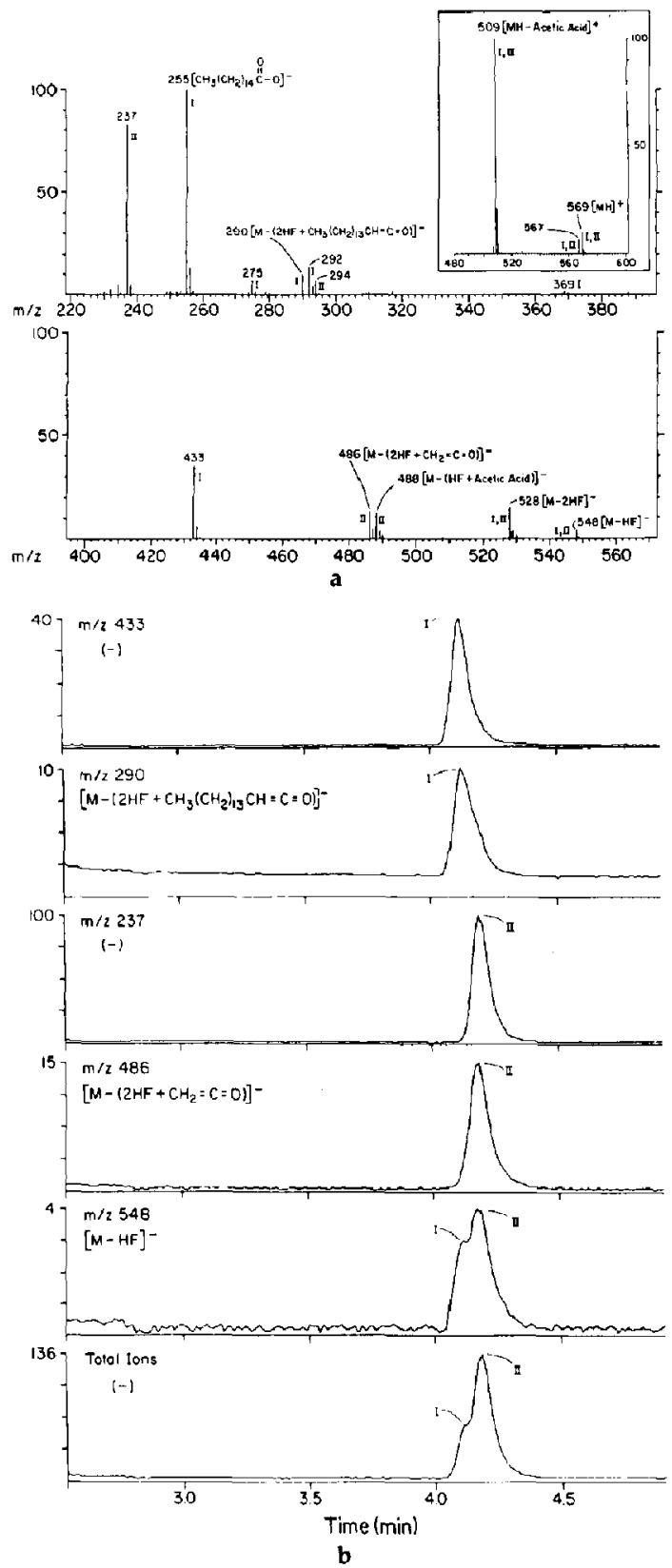

Figure 3. GC/MS andlysis of the HFB derivative of C16:0AGPC. A $40-n g$ aliquot from a $2-\mu \mathrm{g}$ derivatization was injected into the GC. (a) The EC mass spectrum is displayed in the larger trace, with the PICI spectrum included as an insert. The spectra represent a summation across both closely eluting GC peaks, with notations of $I$ and II indicating association of an ion with a particular GC peak. (b) Selected ion retrieval traces for the GC/EC-MS analysis-here the association of ions at $m / z$ and 290 with $G C$ peak $I$ is contrasted with corresponding ions at $m / z 237$ and 486 in peak II. 
ion retrieval traces in Figure $3 \mathrm{~b}$. With the DB-1 column used in the present study it was not possible to separate the GC peaks of C16:0-AGPC/HFB sufficiently to be able to obtain representative spectra of each pure component. For comparison, the $\mathrm{CI}$ spectra and selected ion retrieval traces for C16:0AGEPC/HFB are presented in Figure 4.

Structures for ions such as $m / z 548,528,488$, and 486, as shown in Figure 3a, could be easily assigned, based on losses of HF, ketene, and/or acetic acid. (Note that the loss of 82 mass units can either represent a combination of ketene and HF or a molecule of acetyl fluoride. The latter is thermodynamically favored, but because $\mathrm{HF}$ and ketene are individually lost in the formation of other ions in the spectrum, these moieties are represented separately in our spectral interpretation.) It was further evident that the ion at $m / z 255$ represented the 16-carbon carboxylate
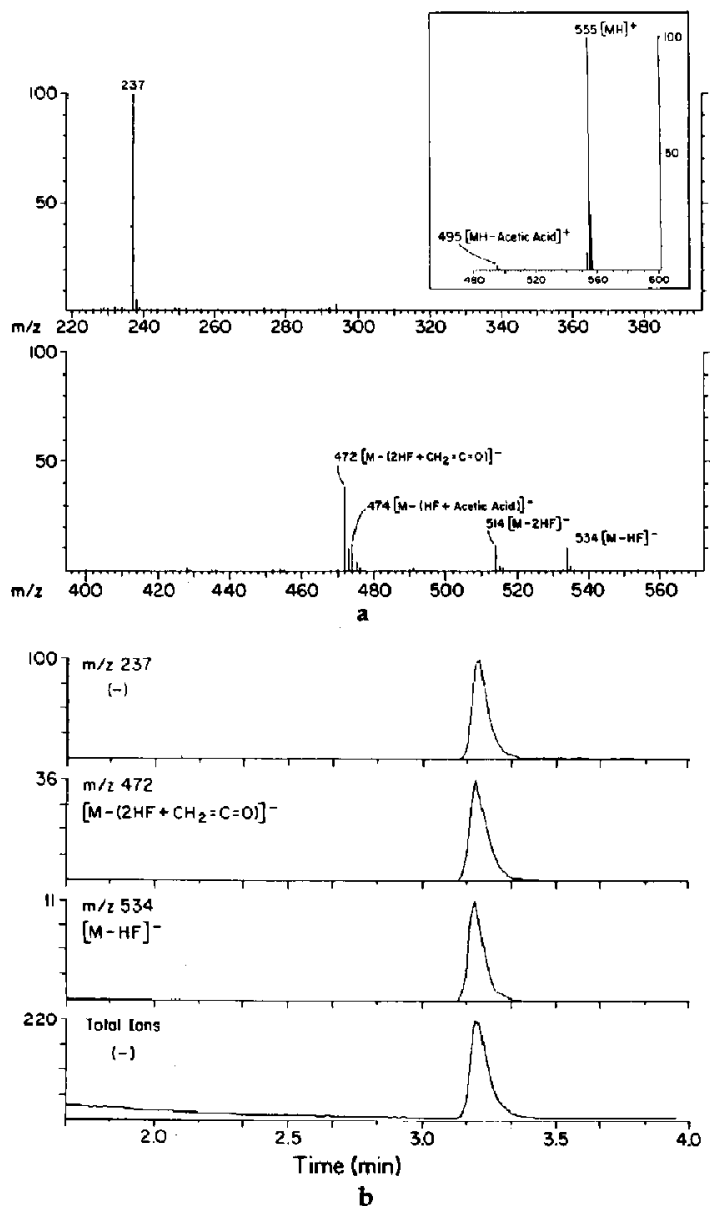

Figure 4. GC/MS analysis of the HFB derivative of C16:0AGEPC. A 40-ng aliquot from a $2-\mu \mathrm{g}$ derivatization was injected into the GC. (a) The EC mass spectrum is displayed in the larger trace, with the PICI spectrum included as an insert. (b) Selected ion retrieval traces for the GC/EC-MS analysis. anion, by comparison with the spectrum of C18:0AGPC/HFB, which exhibited an analogous ion at $\mathrm{m} / z 283$ (data not shown). Elucidation of the intense ions at $m / z 237\left([\mathrm{M}-331]^{-}\right)$and $m / z 433\left([\mathrm{M}-135]^{-}\right)$ was initially unclear. Evaluation of the EC mass spectrum of the $\left[{ }^{13} \mathrm{C}_{2}\right]$ acetate analogue of $\mathrm{C} 16: 0-\mathrm{AGPC} /$ HFB, shown in Figure 5, confirmed that the formation of the ions at $m / z 488$ and $m / z 486$ involved the loss of the two ${ }^{13} \mathrm{C}$ atoms. On the other hand, because the peak at $\mathrm{m} / \mathrm{z} 237$ shifted to $\mathrm{m} / \mathrm{z} 239$ in the labeled compound, this ion must retain the labeled acetyl moiety. Furthermore, the ion at $m / z 433$ was found to increase by 28 mass units to $m / z 461$ in the negative ion spectrum of C18:0-AGPC/HFB (data not shown), indicating that the fatty acyl group was included in this fragment.

Accurate mass measurements of the EC-MS ions at $m / z 237$ and 433 were then obtained. The results are presented in Table 1. Tandem mass spectrometric analyses were also performed; the product ion MS/MS spectra for the ions at $m / z 237$ and 433 are shown in Figure 6. Based on these results it was possible to postulate the following general structure for the ions at $\mathrm{m} / z 237$ and 433 (Figure 7).

Subsequent MS/MS experiments indicated that the ions at $\mathrm{m} / \mathrm{z} 237$ and 433 had no detectable precursors, strongly implying that these ions were formed by a concerted mechanism immediately after EC ionization. The proposed formation of these ions is given in Scheme I. (Note that for clarity, the mechanism is depicted in a stepwise manner; the intermediate ions shown in Scheme I were not individually detected.) The proposed fragmentation pathway bears some analogy to the site-specific rearrangement of pentafluoropropionyl derivatives of $\beta$-hydroxyamines reported by Low and Duffield [9], in which a similar fluorine atom expulsion was observed.

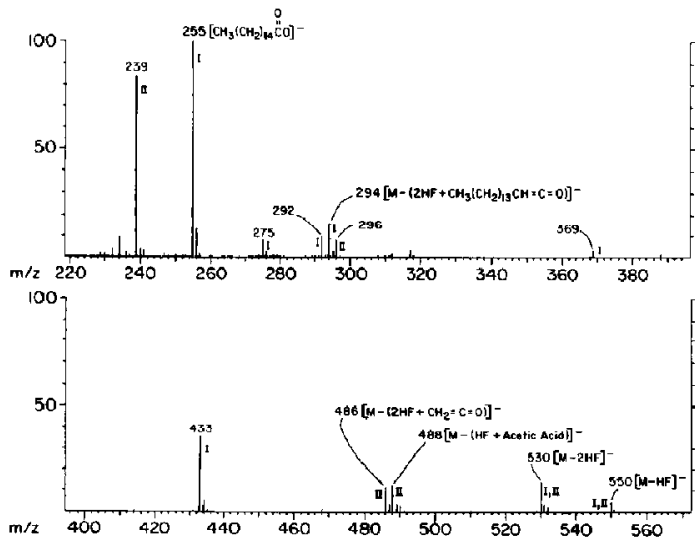

Figure 5. EC mass spectrum of the HFB derivative of C16:0$\left[{ }^{13} \mathrm{C}_{2}\right.$ ] acetyl-GPC. Please note that the ${ }^{13} \mathrm{C}$ labels have been omitted for the structural assignment of $\mathrm{m} / \mathrm{z} 486$ and 488 to enhance readability. 
Table 1. Accurate mass measurement of ions at $m / z 237$ and 433 in the EC mass spectrum of $\mathrm{C} 16: 0-\mathrm{ACPC} / \mathrm{HFB}$

\begin{tabular}{llc}
\hline Measured mass & Formula & $\begin{array}{c}\text { Error } \\
\text { (mmu) }\end{array}$ \\
\hline 236.99929 & $\mathrm{C}_{6} \mathrm{H}_{3} \mathrm{O}_{3} \mathrm{~F}_{6}$ & +0.6 \\
433.21739 & $\mathrm{C}_{20} \mathrm{H}_{31} \mathrm{O}_{3} \mathrm{~F}_{6}$ & -0.4 \\
\hline
\end{tabular}

A summary of the MS/MS data is presented in Figure 8 . The mass value for the molecular anion (not detected) is shown by the dotted line. The arrows and adjacent horizontal bars drawn directly under the dotted line represent ions formed from the molecular anion. The length of each bar is proportional to that ion's relative intensity in the EC mass spectrum. Formation of product ions is shown by the second level of arrows and horizontal bars. The length of each of these bars represents the relative intensity of each ion in the product ion scan.

The product ion MS/MS spectra also showed that the ions at $m / z 195$ and 175 were formed from both $m / z 237$ and 433. In addition, the ion at $m / z 195$ was found to be a prerursor of $\mathrm{m} / \mathrm{z} 175$ (data not shown). Proposed mechanisms for the formation of these ions are presented in Schemes II and III.

\section{Conclusions}

The results of this study demonstrate the presence of two positional isomers in the HFB-derivatized samples of AGPC. The earlier of the two closely eluting
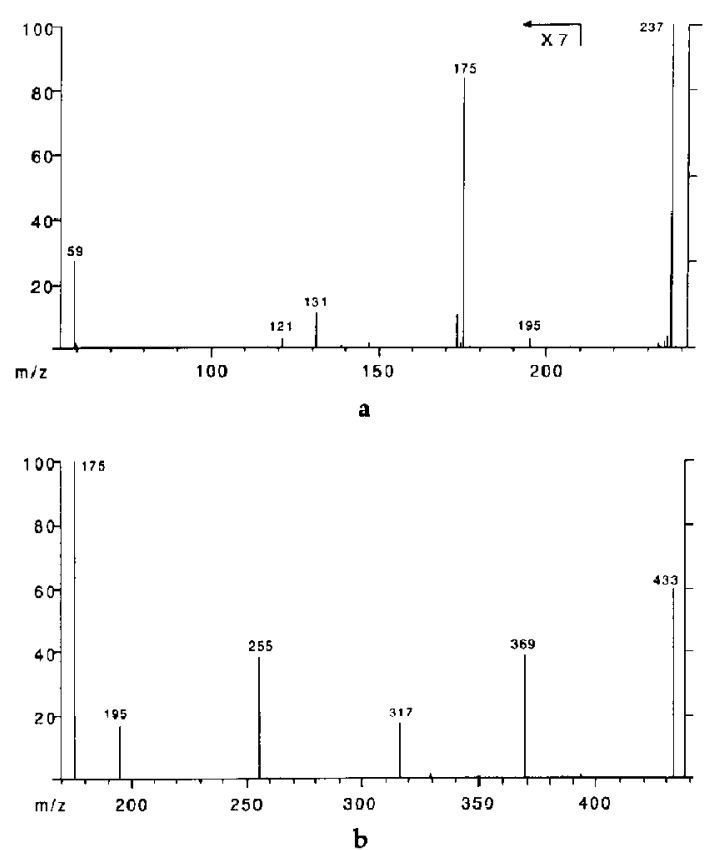

Figure 6. Product ion MS/MS spectra for ions at (a) $\mathrm{m} / \mathrm{z} 237$ and (b) $m / z 433$ formed in the EC-MS analysis of C16:0AGEPC/HFB

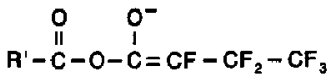

$$
\begin{aligned}
& \mathbf{R}^{\prime}=\mathrm{CH}_{3} \quad \mathrm{~m} / \mathrm{z} 237 \\
& R^{\prime}=\mathrm{CH}_{3}\left(\mathrm{CH}_{2}\right)_{14} \quad \mathrm{~m} / \mathrm{z} 493
\end{aligned}
$$

Figure 7. Proposed structure of ions at $m / z 237$ and 433 in the EC mass spectrum of C16:0-AGPC/HFB.

GC peaks represents the 1-acetyl-2-long chain acyl isomer, based on interpretation of the intense [M$135]^{-}$ion in the mass spectrum of peak 1 . This ion is formed by a rearrangement-reaction between the acyl group on carbon- 2 of glycerol and the HFB moiety on carbon-3. In an analogous manner, peak II represents the 1-long chain acyl-2-acetyl isomer characterized by the ion at $m / z 237$. In support of this conclusion, an ion at $m / z 237$ is also observed in the $\mathrm{EC}$ mass spectrum of AGEPC/HFB homologues (as demonstrated in Figure 4 for (16:0-AGEPC), where isomerization between positions 1 and 2 is not possible. Previous studies have shown that substituent exchange between positions 2 and 3 on glycerol does not occur during direct derivatization [3], and the present results agree with those findings. If a 1-acyl-2HFB-3-acetyl-glycerol were formed in the reaction, it would be expected that both rearrangement ions $(\mathrm{m} / \mathrm{z}$ 237 and 433 ) would be observed in the corresponding EC spectrum. However, the ions at $m / z 433$ and 237 were exclusive to GC peaks I and II, respectively, giving additional proof that exchange between positions 2 and 3 on the glycerol does not occur.

Efforts are currently underway to deduce the origin of the isomerization in the acyl analogues. Earlier studies have shown that direct HFB derivatization of AGEPC proceeds without the isomerization seen after HF or phospholipase C hydrolysis [3]. Furthermore, preliminary nuclear magnetic resonance studies have indicated that the starting lyso-GPC contained two isomers prior to acetylation and derivatization, in agreement with the findings of Pluckthun and Dennis [10].

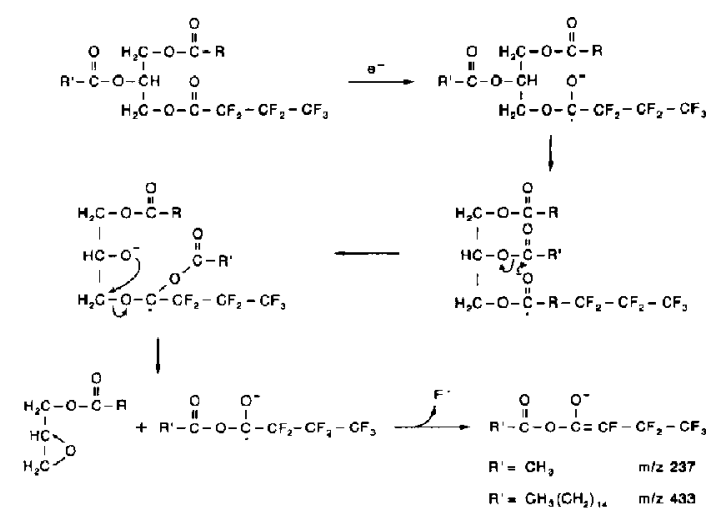

Scheme I 


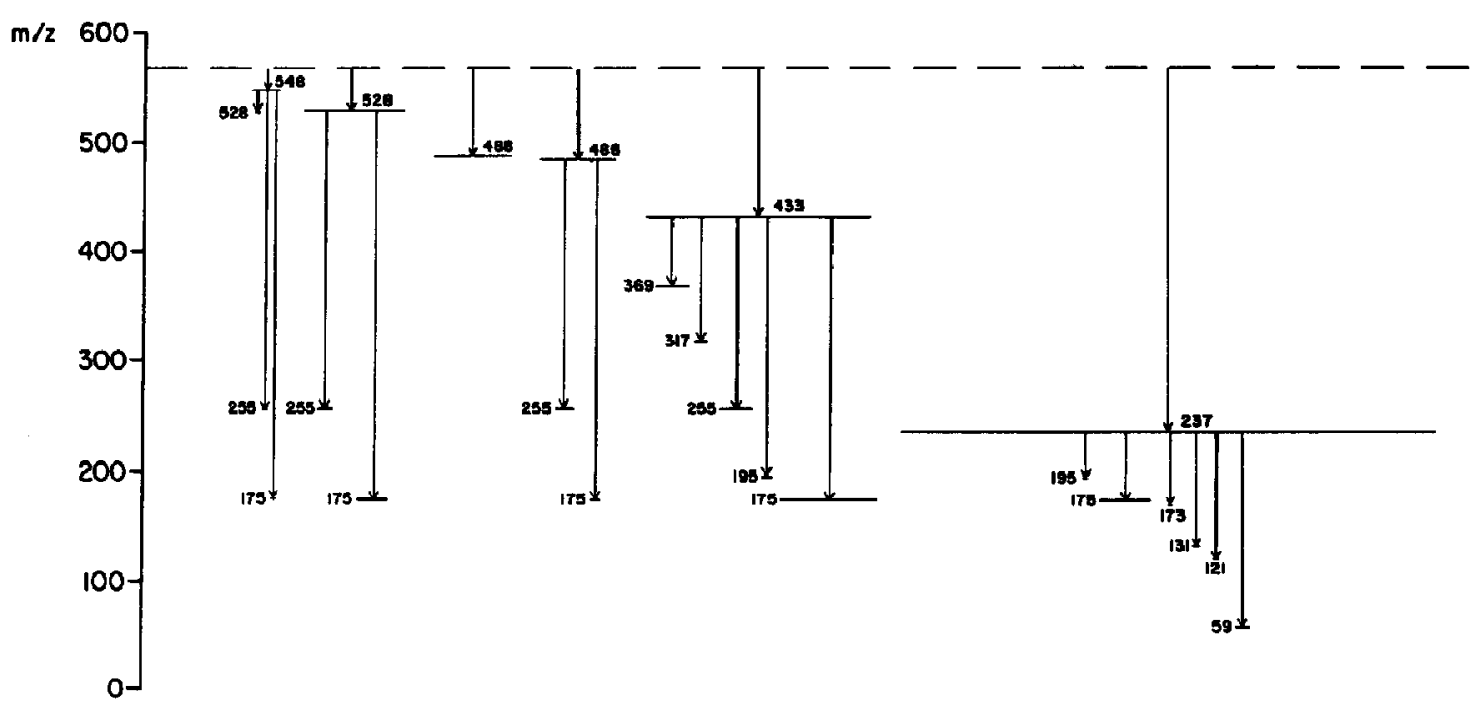

Figure 8. Summary of selected ions in EC MS/MS analysis of C16:0-AGPC/HFB.

The distinctive fragmentation of the HFB derivative of AGPC and AGEPC is of immense value in structural elucidation of samples of biological origin. Although other GC/MS methods may be capable of providing comparable or better sensitivity, they do not yield the degree of specific structural information afforded by EC-MS of the HFB derivative. For example, exceedingly low detection limits (subpicogram) have been reported for EC-MS analysis of the PFB derivative of AGEPC [11]. However, because the mass spectrum of this derivative is comprised predominantly of $[\mathrm{M}]$, this type of analysis only furnishes information about the molecular weight of each analyte. This presents a serious problem for samples which contain both acyl- and alkyl-PAF because, for example, C16:0-AGPC/PFB is isobaric with C17:0AGEPC/PFB, and both C16:0-AGPC and C17:0AGEPC have been shown to be produced by stimulated human neutrophils $[4,8]$. On the other hand, with HFB derivatives one can easily distinguish between acyl- and alkyl-PAF. For 1-acyl-2-acetyl-3-HFBglycerols, the ions representing $[\mathrm{M}-82]^{-}$and $[\mathrm{M}-80]^{-}$ are equally intense, whereas the former ion is signifcantly more intense than the latter one for HFB derivatives of comparable 1-O-alkyl-2-acetyl compounds. Furthermore, EC-MS analysis of PAF/HFB

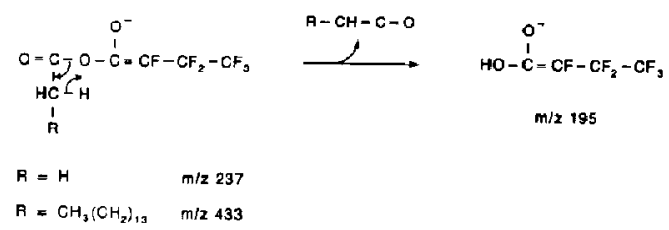

Scheme II derivatives yields specific information about the position of substituents on the glycerol backbone. The presence of a 2-acetyl group on a PAF analogue is indicated by an ion at $m / z 237$. If the acetyl group is attached to carbon- 1 and a different acyl group is esterified at the 2-position, the appropriate carboxylate anion for the substituent at carbon- 2 and an ion representing $[\mathrm{M}-135]^{-}$is observed. Because the $s n-2$ acetyl group is essential for the potent biological activity of this class of phospholipids, EC-MS analysis of HFB derivatives provides an extremely powerful tool to screen for candidate PAF molecules in biologically active samples of unknown composition.

\section{Acknowledgments}

This study was supported in part by National Institutes of Health (NIH) grants HL-22555 and AI-21818. Accurate mass measurements and MS/MS analyses were performed at the Michigan State University Mass Spectrometry Facility, which is

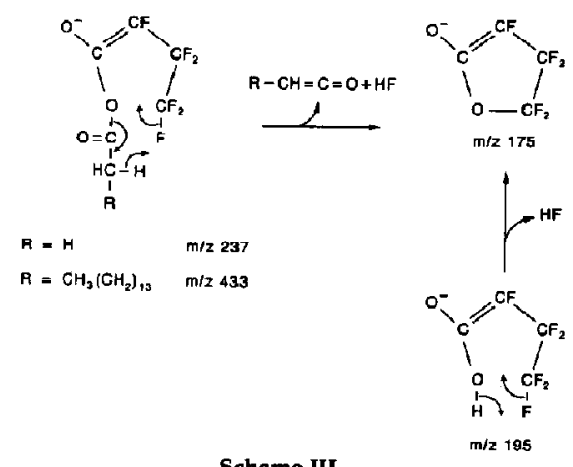

Scheme III 
supported in part by NIH grant DRR-00480. The format for the MS/MS data presented in Figure 8 is styled after the "TREE" program of Finnigan-MAT. The authors are grateful to Professor Charles DePuy for his advice on negative ion fragmentation mechanisms.

\section{References}

1. Yinckard. R. N.; Ludwig, J. C.; McManus, L. M. In Inflammation: Basic Principles and Clinical Correlates; Gallin, J. I.; Goldstein, I. M.; Snyderman, R., Eds.; Raven: New York, 1988; pp 139-167.

2. Sturk, A.; Schaap, M. C. L.; Prins, A.; Wouter ten Cate, J.; van den Bosch, H. Biochim. Biophys. Acta 1989, 993, 148-156.

3. Satsangi, R. K.; Ludwig, J. C.; Weintraub, S. T.; Pinckard, R. N. J. Lipid Res. 1989, 30, 929-937.
4. Weintraub, S. T.; Lear, C. L.; Pinckard, R. N. /. Lipid Res, 1990, 31, 719-725.

5. Bligh, E. G.; Dyer, W. J. Can. J. Biothem. Physiol. 1959, 37, 911-917.

6. Demopoulos, C. A.; Pinckard, R. N.; Hanahan, D. J. J. Biol. Chem. 1979, 254, 9355-935R.

7. Bartlett, G. I. Biol. Chem. 1959, 234, 466-468.

8. Weintraub, S. T.; Ludwig, J. C.; Mott, G. E.; McManus, L. M.; Pinckard, R. N. Biochem. Biophys. Res. Commun. 1985 $129,868-876$

9. Low, G. K,-C.; Duffield, A. M. Biomed. Mass Spectrom, 1984, $11,177-181$.

10. I'luckthun, A.; Dennis, E. A. Brochemistry 1982, 2I, $1743-1750$.

11. Ramesha, C. S; Pickett, W. C. Biomed. Mass. Spectrom. 1986, 13, 107-111. 